\title{
High-flow nasal cannula compared with conventional oxygen therapy for acute hypoxemic respiratory failure: author's reply
}

\author{
Bram Rochwerg ${ }^{1,2,7^{*}}$ (D, D. Granton ${ }^{1}$, D. X. Wang ${ }^{3}$, S. Einav ${ }^{4,5}$ and K. E. A. Burns ${ }^{6}$
}

(C) 2019 Springer-Verlag GmbH Germany, part of Springer Nature

Dear Editor,

Thanks to Dr. Luo and colleagues for their letter and comments [1]. In the protocol for our systematic review, we decided a priori that all analysis would be conducted using a random effects model, as this analytic plan better accounts for between-study differences [2]. In situations where statistical heterogeneity is minimal, the output from random effects models closely matches that of fixed effects models. However, in response to this letter, we did perform fixed effects analysis for this outcome and the pooled point estimate and $95 \%$ confidence intervals do not change.

We focused our TSA analysis on the outcomes most likely to be considered for future RCTs. As escalation of therapy is a composite, including outcomes of variable clinical and patient importance, we intentionally highlighted 'need for IMV' and 'mortality'. For the TSA analysis, we had initially used the relative risk reductions from our pooled analysis to inform the sample size calculation. After peer review of the manuscript, we were asked to choose a threshold consistent with what we believed would be a clinically important difference. As such, we selected a $15 \%$ relative risk reduction for this dichotomous outcome.
Medical Center, Jerusalem, Israel. ${ }^{5}$ Faculty of Medicine, Hebrew University, Jerusalem, Israel. ${ }^{6}$ Interdepartmental Division of Critical Care Medicine, University of Toronto, Toronto, ON, Canada. ${ }^{7}$ Division of Critical Care, Department of Medicine, Juravinski Hospital, 711 Concession St, Hamilton, ON L8V 1C1, Canada.

\section{Publisher's Note}

Springer Nature remains neutral with regard to jurisdictional claims in published maps and institutional affiliations.

Accepted: 23 May 2019

Published online: 24 June 2019

\section{References}

1. Luo MS, Huang GJ, Wu L (2019) High flow nasal cannula compared with conventional oxygen therapy for acute hypoxemic respiratory failure. Intensive Care Med. https://doi.org/10.1007/s00134-019-05652-8

2. Rochwerg B, Granton D, Wang DX et al (2019) High flow nasal cannula compared with conventional oxygen therapy for acute hypoxemic respiratory failure: a systematic review and meta-analysis. Intensive Care Med 45(5):563-572. https://doi.org/10.1007/s00134-019-05590-5 (published online first: Epub date)

\section{Author details \\ ${ }^{1}$ Department of Medicine, McMaster University, Hamilton, ON, Canada. \\ ${ }^{2}$ Department of Health Research Methods, Evidence and Impact, McMaster University, Hamilton, ON, Canada. ${ }^{3}$ Schulich School of Medicine, Western \\ University, London, ON, Canada. ${ }^{4}$ General Intensive Care Unit, Shaare Zedek}

*Correspondence: rochwerg@mcmasterca

7 Division of Critical Care, Department of Medicine, Juravinski Hospital, 711 Concession St, Hamilton, ON L8V 1C1, Canada

Full author information is available at the end of the article

\section{Springer}

